\title{
"Crossed" somatoparaphrenia: an unusual new case and a review of the literature
}

\author{
Fabienne Perren · Lukas Heydrich • Olaf Blanke • \\ Theodor Landis
}

Received: 12 December 2013 / Accepted: 6 September 2014 / Published online: 10 October 2014

(C) Springer-Verlag Berlin Heidelberg 2014

\begin{abstract}
Somatoparaphrenia is a delusional misidentification and confabulation of body parts, usually arm or hand, opposite to a cerebral lesion, generally of the "minor" right hemisphere. There is some controversy concerning lesion site (fronto-parietal; parieto-temporal; posterior insula, additional subcortical nuclei) or necessary associated symptoms (hemiparesis/plegia, anosognosia, neglect, position sense deficit). We here present a patient who is unusual in many respects, that is: (1) he is a right-hander with somatoparaphrenia after a "dominant" left-hemisphere lesion associated with aphasia and ideomotor apraxia, but also with right hemineglect. He thus has "crossed" somatoparaphrenia; (2) his delusional misidentification concerned the right leg and not the arm or hand; (3) he has no anosognosia; (4) his proprioception is disturbed for the leg only; and (5) the lesion site is very posterior, a left occipito-parietal haemorrhage without involvement of the frontal lobe or the posterior insula. We present this case together with the seven other cases of "crossed somatoparaphrenia" with and without aphasia we found since 1935 in the literature and discuss their relevance in relation to the above controversies.
\end{abstract}

F. Perren $(\bowtie) \cdot$ L. Heydrich $\cdot$ O. Blanke $\cdot$ T. Landis Department of Neurology, HUG, University Hospital and Medical Faculty of Geneva, Rue Gabrielle Perret-Gentil 4, 1211 Geneva 14, Switzerland

e-mail: fabienne.perren@hcuge.ch

L. Heydrich · O. Blanke

Laboratory of Cognitive Neuroscience, Brain-Mind Institute, Faculty of Life Sciences, Ecole Polytechnique Fédérale de

Lausanne (EPFL), 1015 Lausanne, Switzerland

O. Blanke

Center for Neuroprosthetics, Ecole Polytechnique Fédérale de Lausanne (EPFL), 1015 Lausanne, Switzerland
Keywords Somatoparaphrenia $\cdot$ Left brain damage · Right hemineglect · Anosognosia · Position sense · Cerebral laterality

\section{Introduction}

Somatoparaphrenia is a delusional belief concerning the controlateral side of the body in brain-damaged patients. This term has been introduced by Gerstmann (1942) to describe "illusions or distortions concerning the perception of and confabulations or delusions referring to the affected limbs or side". It is commonly associated with extensive "minor" right brain lesions; especially the right parietal and parieto-temporal junction area is an important structure, which, when damaged, can lead to body perception disturbances, neglect and anosognosia. It has, however, been postulated that for somatoparaphrenia to occur, extension of this "core" lesion into additional structures is needed. The three different additional structures identified by different investigators are as follows: (1) the posterior insula (Baier and Karnath 2008), (2) the right orbito- and medial frontal lobe (Feinberg et al. 2010) and (3) subcortical nuclei (Gandola et al. 2012).

Moreover, different studies have found different symptoms that were highly associated with the presence of somatoparaphrenia, such as: (1) hemiparesis/plegia (Vallar and Ronchi 2009; Baier and Karnath 2008), (2) anosognosia (Baier and Karnath 2008), (3) position sense deficit (Vallar and Ronchi 2009) and (4) neglect (Vallar and Ronchi 2009; Baier and Karnath 2008; Feinberg et al. 2010; Gandola et al. 2012).

In their exhaustive review, Vallar and Ronchi report 56 cases of which in only four (7.1\%) right-handed patients' right-sided body parts were involved (Schilder 1935; 
Nielsen 1938; Miura et al. 1996; Schiff and Pulver 1999). One might call these cases "crossed somatoparaphrenia", in analogy to "crossed aphasia". Somatoparaphrenia is a rare symptom. In an analysis of 79 consecutive patients with right-brain stroke and left hemiparesis/hemiplegia, Baier and Karnath (2008) found six patients who demonstrated somatoparaphrenia $(7.5 \%)$. If one considers that only $7.1 \%$ patients of the largest literature review on somatoparaphrenia (Vallar and Ronchi 2009) had "crossed somatoparaphrenia", this would mean a prevalence of $0.5 \%$ for the latter, which is roughly in the same order of magnitude than the prevalence of "crossed aphasia" given in the stroke literature of 0.4-3.5\% (Dewarrat et al. 2009).

Here, we present an unusual new case of "crossed somatoparaphrenia", and add three more patients we found in the literature (Cohen et al. 1991; Beato et al. 2010; Cogliano et al. 2012). We will subsequently compare the characteristics of these 8 "crossed somatoparaphrenia" patients with the remaining 52 "non-crossed" cases recently reviewed (Vallar and Ronchi 2009).

\section{Case report}

This 72-year-old right-handed retired architect, known and treated for hypertension and hypercholesterolaemia, suffered while driving his car a feeling of faintness without loss of consciousness followed by an acute state of agitation, language difficulties and weakness of his right arm and leg. At admission, the patient was severely aphasic, with fluent paraphasic incoherent speech and severe oral comprehension deficit. He showed severe right-sided hemineglect, a right homonymous hemianopia and a right hemiparesis of arm and leg. Sensory examination was not possible.(NIH Stroke Scale of 12-14). Brain CT (Fig. 1a, b) and MRI (Fig. 1c, d) showed an acute parenchymal haemorrhagic lesion in the left parieto-occipito-temporal region with a moderate mass effect on the temporal horn of the left ventricle.

He recovered rapidly during the next 5 days. This was the time during which the somatoparaphrenia episodes happened. He spontaneously complained (pointing to his right
Fig. 1 CTscan (a, b) MRI (c, d) Acute parenchymal haemorrhagic lesion in the left parieto-occipito-temporal region with a moderate mass effect on the temporal horn of the left ventricle
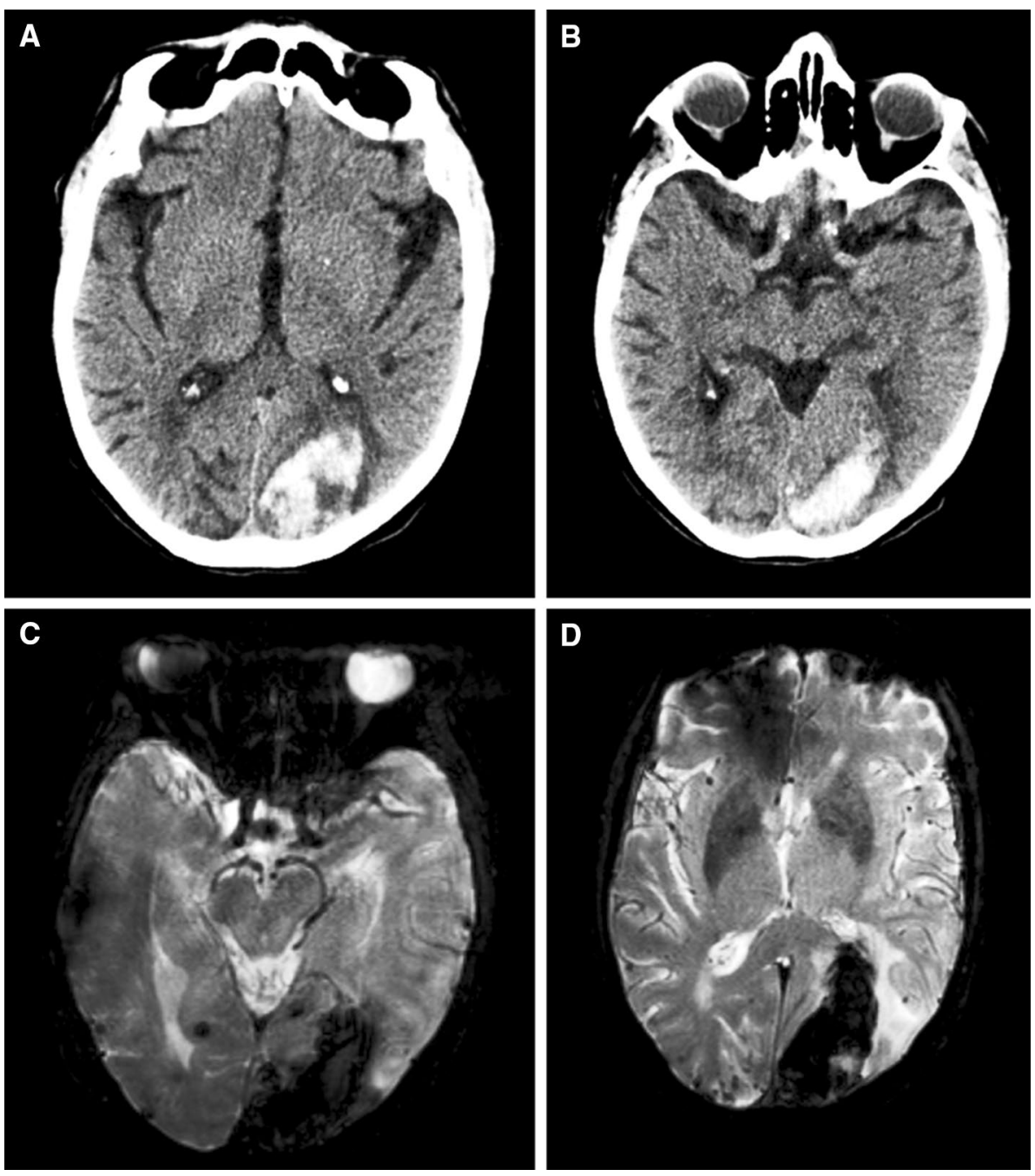
leg) about having something like "a piece of wood" or "a thing" in his bed. On other occasions, he also claimed that it was "the leg of somebody else" that he did not know. Upon questioning, he said that he did not feel it and that the form was somehow changed and that he could not move foot and toes. Inspection of the leg in a mirror did not modify these phenomena. He also was frequently seen hitting his right leg and handled it as he would like to get rid of it (misoplegia). After permission by the patient and under his observation, one of the examiners placed his right leg in the patient's bed under the cover beside the patient's right leg. When only exposing the examiner's leg, the patient misidentified it as his own leg. When the examiner exposed the examiner's leg and moved it, the patient identified it correctly as belonging to the examiner. There were no spontaneous complaints about the right upper limb; upon questioning the patient identified his right arm as belonging to him. While the procedure with his leg was done only on two consecutive days, hand identification was done routinely during every ward round. Specifically asked for he said: something is different with this right hand. If I compare it with my left its too big, too dry, I cannot feel it correctly, I do not know what happened, it might not be mine, but when I look at it, it looks normal. His right hand was every day shown to him in his attended left visual hemispace, either by moving it across his body midline to the left, or by his head turned to the right that the head centred axis would pass at the right of his right hand. He would identify it as belonging to him. When shown together with the doctor's hand, he would hesitate but finally correctly identify it as his own hand.

Sensory exam became possible. There was a mild right hemihypoesthesia more marked for the leg. Position sense was normal on the right hand but impaired on the right foot. Right hemiparesis improved more for the arm than the leg. He spontaneously moved both the right arm and leg but frequently failed on verbal request. He was neither confused nor disoriented. He recovered rapidly from his aphasia. Spontaneous speech became normal except for some word finding difficulties and occasional paraphasias. Oral comprehension strongly improved but there remained some impaired text comprehension and severe ideo-motor apraxia. He was unable with either hand to pantomime gestures on verbal command but was equally unable to do so when the gestures were shown to him. However, he correctly performed the gestures when given the object. There was no agnosia. He recognized illusory contours from a set of Kanizsa figures, he recognized images from defragmented figures of a set of Street figures, he recognized objects from a set of unusual (non-canonical) views, and there was no prosopagnosia or visual associative agnosia.

There was no anosognosia. When asked why he was in the hospital, he said "I had a stroke". When asked what really happened, he said "something happened to my right arm and leg".

He had a dense homonymous hemianopsia to the right but also a right visual extrapersonal neglect. He tended to keep his head turned to the left and did not make spontaneous saccades into the right hemispace. There were no exploratory saccades when a stimulus was moved into the right hemispace. He omitted to bisect most lines on the right on a line bisection task and of the few realized lines to the right of the midline mark was displaced to the left. There was no right auditory extinction on double simultaneous auditory stimulation. There was mild personal neglect. He would almost always extinguish right-sided touch on double simultaneous stimulation but at occasions there was sensory misplacement from the hand to the ipsilateral face (allesthesia) or even to the opposite side (allochiria). He usually acknowledged when only the right hemibody was touched. His capacity to point with his left hand to his right arm or leg was fluctuating. At times, he pointed to his right hand crossing the body midline with the left hand on request.

By day 6 , he became drowsy and later comatose (GSC 6/15) subsequent to an oedema of his haemorrhage with mass effect and he later died from medical complications.

\section{Discussion}

Brain damage in cases of "crossed somatoparaphrenia"

The different lesion locations of 8 cases of crossed somatoparaphrenia are summarized in Table 1. Three associated lesion sites, the medial and orbito-frontal lobe (Feinberg et al. 2010), the posterior insula (Baier and Karnath 2008) and subcortical nuclei (Gandola et al. 2012) are of particular interest since they have been reported as being "crucial" for the occurrence of somatoparaphrenia. In summary, in two cases, there is no precise lesion anatomy mentioned (Schilder 1935; Nielsen 1938), while in the remaining six cases all had temporo-parietal pathology. The question of whether the posterior insula was involved cannot be answered in two cases (Miura et al. 1996; Beato et al. 2010), but the posterior insula was spared in two cases (Cogliano et al. 2012; present case), while in all others involvement of subcortical nuclei and/or posterior insula are quite likely. Frontal structures were completely spared in our case, and the case of Cogliano et al. (2012) and medial frontal structures were very likely not involved in the cases of Miura et al. (1996), Beato et al. 2010), Schiff and Pulver (1999).

In the cases of "crossed somatoparaphrenia", none of the postulated additional lesions sites appears to be crucial; since there are cases in which none of these sites are involved despite the presence of somatoparaphrenia. 


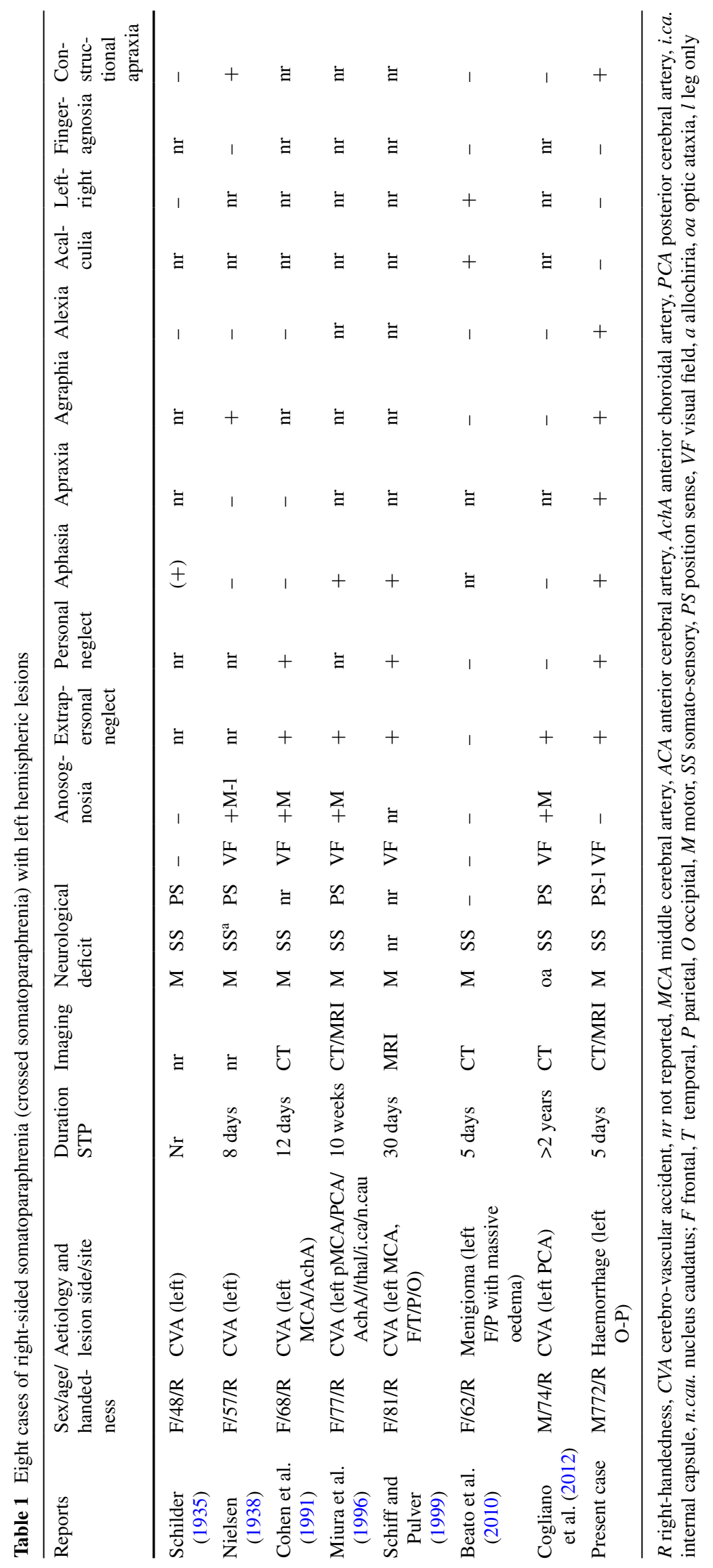


Associated symptoms in "crossed somatoparaphrenia"

Three symptoms, neglect, anosognosia and a position sense deficit are of particular interest since they have been associated "crucially" with the occurrence of somatoparaphrenia (STP) by different authors (Vallar and Ronchi 2009; Baier and Karnath 2008; Feinberg et al. 2010) (see Table 1). In the eight cases of crossed somatoparaphrenia, when reported, somatosensory deficit was present in $100 \%$ (STP $90 \%$ ), hemiparesis was present in $87.5 \%$ (STP $96 \%$ ), position sense deficits in $83 \%$ (STP $96 \%$ ), visual field deficit in $71 \%$ (STP $88 \%$ ), extrapersonal neglect in $83 \%$ (STP $94 \%$ ), personal neglect in $60 \%$ (STP $88 \%$ ) and anosognosia for hemiparesis/plegia in $57 \%$ (STP $83 \%$ ).

Thus, somatosensory deficit, hemiparesis, deficient position sense, visual field deficit and extrapersonal neglect were as frequent or almost as frequent in crossed than in uncrossed cases, while personal neglect and anosognosia for hemiparesis/plegia were about $25 \%$ less frequent in crossed than in uncrossed cases. Altogether in 5 patients (Nielsen 1938; Miura et al. 1996; Schiff and Pulver 1999; Beato et al. 2010; present case), language-related symptoms coexisted with somatoparaphrenia.

Unusual associated symptoms in crossed somatoparaphrenia comprise: lack of paresis but severe right-sided optic ataxia (Cogliano et al. 2012), somatoparaphrenia for the leg, but not the arm (present case, to our knowledge only reported once before by Barkman (1925), hyperfamiliarity for faces (Schilder 1935) (recently associated with left (dominant) temporo-occipital lesion (Vuilleumier et al. 2003).

\section{Conclusions}

Somatoparaphrenia is a symptom highly associated with hemispheric specialization. It occurs almost exclusively after lesions of the "minor" right hemisphere. "Crossed" somatoparaphrenia is rare, as rare as "crossed" aphasia. Although the very small number of such cases does not permit strong conclusions, in left brain damage, somatoparaphrenia is less strongly associated with several previously postulated "crucial" lesion sites such as the posterior insula, the orbito- and medial frontal lobes and subcortical nuclei than somatoparaphrenia after right brain damage. This means that the occurrence of somatoparaphrenia as a symptom is not crucially dependent upon the involvement of these sites. Crossed somatoparaphrenia is also less strongly associated with anosognosia and personal neglect than somatoparaphrenia after right brain damage. However, crossed somatoparaphrenia remains equally strongly associated with deficient position sense and extrapersonal neglect as well as with hemiparesis and hemihypoesthesia than somatoparaphrenia after right brain damage, despite the co-existence of language dominance in the same hemisphere. One could thus argue that somatoparaphrenia relies upon a combination of extrapersonal neglect (typically due to non-dominant lesions, but in our and two other cases associated with language dominant lesions), intact verbal capacities to the extent that the patient can produce "illusional, confabulatory or delusional ideas of a peculiar nature", and a sensory-motor hemisyndrome which includes a position sense impairment.

Acknowledgments This work has been supported by the Swiss National Science Foundation Grant No. 320030-132967.

\section{References}

Baier B, Karnath HO (2008) Tight link between our sense of limb ownership and self-awareness of actions. Stroke 39:486-488

Barkman A (1925) De l'anosognosie dans l'hémiplégie cérébrale: contribution Clinique a l'étude de ce symptôme. Acta Med Scand 52:235-254

Beato R, Martins W, Nicolato A, Ulhoa TH, Oliveira MM, Freitas e Avelar IF (2010) Transitory somatoparaphrenia associated with a left frontoparietal menigioma. J Neurol 257:1208-1210

Cogliano R, Crisci C, Conson M, Grossi D, Trojano L (2012) Chronic somatoparaphrenia: a follow-up study on two clinical cases. Cortex 48:758-767

Cohen L, Rémy P, Leroy A, Gény C, Degos JD (1991) Minor hemisphere syndrome following left hemispheric lesion in a right handed patient. JNNP 54:842-843

Dewarrat GM, Annoni JM, Fornari E, Carota A, Bogousslavsky J, Maeder P (2009) Acute aphasia after right hemisphere stroke. J Neurol 256:1461-1467

Feinberg TE, Venneri A, Simone A, Fan Y, Northoff G (2010) The neuroanatomy of anosognosia and somatoparaphrenia. J Neurol Neurosurg Psychiatry 81:276-281

Gandola M, Invernizzi P, Sedda A, Ferré ER, Sterzi R, Sberna M et al (2012) An anatomical account of somatoparaphrenia Cortex 48:1165-1178

Gerstmann J (1942) Problem of imperception of disease and of impaired body territories with organic lesions. Arch Neurol Psychiatry 48:890-913

Miura N, Takeda A, Terao S, Tanaka H, Ishikawa S, Mitsuma T (1996) Somatoparaphrenia caused by the lesion in the dominant cerebral hemisphere: a case report. No To Shinkei 48:275-279

Nielsen JM (1938) Gerstmann syndrome: finger agnosia, agraphia, confusion of right and left, and acalculia. Arch Neurol Psychiatry 39:535-560

Schiff ND, Pulver M (1999) Does vestibular stimulation activate thalamocortical mechanisms that reintegrate impaired cortical regions? Proc Biol Sci 266:421-423

Schilder P (1935) The image and appearance of the human body. International Universities Press, New York

Vallar G, Ronchi R (2009) Somatoparaphrenia: a body delusion. A review of the neuropsychological literature. Exp Brain Res 192:533-551

Vuilleumier P, Mohr C, Valenza N, Wetzel C, Landis T (2003) Familiarity for unknown faces after left lateral temporo-occipital venous infarction: a double dissociation with prosopagnosia. Brain 126:889-907 